\title{
A Stable and Convergent Finite Difference Scheme for 2D Incompressible Nonlinear Viscoelastic Fluid Dynamics Problem
}

\author{
Yanhua Cao ${ }^{1}$, Zhendong Luo ${ }^{2, *}$ \\ ${ }^{1}$ School of Sciences, East China Jiaotong University, Nanchang, China \\ ${ }^{2}$ School of Mathematics and Physics, North China Electric Power University, Beijing, China

\section{Email address:} \\ yanhuacao@yeah.net (Yanhua Cao), zhdluo@ncepu.edu.cn (Zhendong Luo) \\ ${ }^{*}$ Corresponding author
}

\section{To cite this article:}

Yanhua Cao, Zhendong Luo. A Stable and Convergent Finite Difference Scheme for 2D Incompressible Nonlinear Viscoelastic Fluid Dynamics Problem. Applied and Computational Mathematics. Vol. 7, No. 1, 2018, pp. 11-18. doi: 10.11648/j.acm.20180701.12

Received: December 4, 2017; Accepted: December 15, 2017; Published: January 12, 2018

\begin{abstract}
In this study, a stable and convergent finite difference (FD) scheme based on staggered meshes for twodimensional (2D) incompressible nonlinear viscoelastic fluid dynamics problem including the velocity vector field and the pressure field as well as the deformation tensor matrix is established in order to find numerical solutions for the problem. The stability, convergence, and errors of the FD solutions are analyzed. Some numerical experiments are presented to show that the FD scheme is feasible and efficient for simulating the phenomena of the velocity and the pressure as well as the deformation tensor in an estuary.
\end{abstract}

Keywords: Finite Difference Scheme, Incompressible Nonlinear Viscoelastic Fluid Problem, Stability and Convergence, Numerical Simulations

\section{Introduction}

Let $\Omega \subset \mathrm{R}^{2}$ be a bounded and connected domain. Consider the following two-dimensional (2D) incompressible nonlinear viscoelastic fluid dynamics problem (see [1]):

$$
\begin{gathered}
\frac{\partial u}{\partial x}+\frac{\partial u}{\partial y}=0, \quad(x, y, t) \in \Omega \times(0, T) \\
\frac{\partial u}{\partial t}+\frac{\partial\left(u^{2}\right)}{\partial x}+\frac{\partial(u v)}{\partial y}+\frac{\partial p}{\partial x} \\
=\mu\left(\frac{\partial^{2} u}{\partial x^{2}}+\frac{\partial^{2} u}{\partial y^{2}}\right)+\frac{\partial\left(F_{1,1}^{2}+F_{1,2}^{2}\right)}{\partial x}+\frac{\partial\left(F_{1,1} F_{2,1}+F_{1,2} F_{2,2}\right)}{\partial y},(x, y, t) \in \\
\Omega \times(0, T), \\
\frac{\partial v}{\partial t}+\frac{\partial(u v)}{\partial x}+\frac{\partial\left(v^{2}\right)}{\partial y}+\frac{\partial p}{\partial y} \\
=\mu\left(\frac{\partial^{2} v}{\partial x^{2}}+\frac{\partial^{2} v}{\partial y^{2}}\right)+\frac{\partial\left(F_{2,1}^{2}+F_{2,2}^{2}\right)}{\partial x}+\frac{\partial\left(F_{1,1} F_{2,1}+F_{1,2} F_{2,2}\right)}{\partial x},(x, y, t) \in \Omega \times
\end{gathered}
$$$$
(0, T) \text {, }
$$

$$
\begin{aligned}
& \frac{\partial F_{1,1}}{\partial t}+\frac{\partial\left(u F_{1,1}\right)}{\partial x}+\frac{\partial\left(v F_{1,1}\right)}{\partial y} \\
= & \frac{F_{1,1} \partial u}{\partial x}+\frac{F_{2,1} \partial u}{\partial y},(x, y, t) \in \Omega \times(0, T), \\
& \frac{\partial F_{1,2}}{\partial t}+\frac{\partial\left(u F_{1,2}\right)}{\partial x}+\frac{\partial\left(v F_{1,2}\right)}{\partial y} \\
= & \frac{F_{1,2} \partial u}{\partial x}+\frac{F_{2,2} \partial u}{\partial y},(x, y, t) \in \Omega \times(0, T), \\
& \frac{\partial F_{2,1}}{\partial t}+\frac{\partial\left(u F_{2,1}\right)}{\partial x}+\frac{\partial\left(v F_{2,1}\right)}{\partial y} \\
= & \frac{F_{1,1} \partial v}{\partial x}+\frac{F_{2,1} \partial v}{\partial y},(x, y, t) \in \Omega \times(0, T), \\
& \frac{\partial F_{2,2}}{\partial t}+\frac{\partial\left(u F_{2,2}\right)}{\partial x}+\frac{\partial\left(v F_{2,1}\right)}{\partial y} \\
= & \frac{F_{1,2} \partial v}{\partial x}+\frac{F_{2,2} \partial v}{\partial y},(x, y, t) \in \Omega \times(0, T),
\end{aligned}
$$


subject to initial conditions

$$
\begin{array}{r}
u(x, y, 0)=u^{0}(x, y), v(x, y, 0)=v^{0}(x, y), \\
F_{l, m}(x, y, 0)=F_{l, m}^{0}(x, y), l, m=1,2,(x, y) \in \Omega,
\end{array}
$$

and boundary conditions

$$
\begin{gathered}
u(x, y, t)=u_{0}(x, y, t), v(x, y, t)=v_{0}(x, y, t), \\
F_{l, m}(x, y, t)=F_{0, l, m}(x, y, t), \\
l, m=1,2,(x, y, t) \in \partial \Omega \times(0, T),
\end{gathered}
$$

where $\boldsymbol{U}=(u, v)^{T}$ represents the unknown fluid velocity, $\boldsymbol{F}=\left(F_{l, m}\right)_{2 \times 2}$ is the unknown deformation tensor, $p$ is the unknown hydrodynamic pressure, $\mu=1 / R e$ is the known coefficients of viscosity, $R e$ represents the Reynolds number, and $u^{0}(x, y), v^{0}(x, y), F_{l, m}^{0}(x, y), u_{0}(x, y, t), v_{0}(x, y, t)$ and $F_{0, l, m}(x, y, t)(l, m=1,2)$ all are given functions.

The 2D incompressible nonlinear viscoelastic fluid dynamics problem, i.e., the system of Eqs (1) - (9) can be used to describe many important physical issues, for example, the complicated hydrodynamic and rheological behaviors of complex fluids, and the electromagnetic behaviors of the materials (see [2-5]). Because the incompressible nonlinear viscoelastic fluid dynamics problem is nonlinear, especially, it does not only contain the velocity vector field as well as the pressure field but also includes the deformation tensor matrix, i.e., includes seven unknown functions, even if References [1,6] had provided the theoretical analysis of existence of generalized solution, finding its analytical solution is a difficult task. One has to rely on numerical solutions.

Though Barton and Kirby have established a finite difference (FD) scheme for fluid flow problems on nonstaggered grids in [7], Khader and Megahed have presented a numerical simulation using the FD method for the flow and heat transfer in [8], and Pandit et al. also have built a transient higher order compact scheme for incompressible viscous flows on geometries beyond rectangular in [9], their problems are different from the system of Eqs (1) - (9). Although Soukup has presented a numerical method based on DGM for solving the system of equations describing motion of viscoelastic fluid with memory in Reference [10], the problem discussed is linear and a transformed Navier-Stokes equation, and the existence and uniqueness as well as the convergence (error estimates) of the numerical solutions had not been studied. Sampaio has also developed some mixed finite element (MEF) models for incompressible viscous flows in References [11, 12], but has yet not provided the theoretical analysis of the existence and uniqueness as well as the convergence (error estimates) of the numerical solutions. Faria and Karam-Filho have also proposed a regularized-stabilized MEF formulation for the steady flow of an incompressible fluid of Bingham type in Reference [13], but their problem is also stationary and linear. Whereas the system of Eqs (1) - (9) here is nonlinear, includes seven unknown functions, and the unknown deformation tensor may be an unsymmetrical matrix, therefore, it is thoughtfully different from those problems in References [7-13] and is more complex than those equations in References [7-13]. Thus, either the establishment of the numerical models or the theoretical analysis of the stability and convergence as well as the error estimates of the numerical solutions for the system of Eqs (1) - (9) includes more difficulties and requires more techniques than those in the above References [7-13], but it is more useful.

Though a stabilized finite volume element (FVE) method for the system of Eqs (1) - (9) has presented in [14], as far as we know, so far, there has not been any report about the FD scheme for the system of Eqs (1)-(9). Compared with the FVE method, the FD scheme for the system of Eqs (1) - (9) can conveniently handle and requires fewer techniques. Therefore, the main task in this article is to construct a stable and convergent FD scheme based on staggered meshes for the system of Eqs (1) - (9) in Section 2, analyze the stability and convergence of the FD solutions for the system of Eqs (1) (9) in Section 3, and offer some experiments to verify that the FD scheme is feasible and efficient for simulating the phenomena of the velocity and the pressure as well as the deformation tensor in an estuary in Section 4. This implies that the work here is improvement and development of the existing those mentioned. Finally, in Section 5, we will summarize some main conclusions.

\section{Establishment of FD Scheme}

Let $\Delta t$ be the temporal step and $\Delta x$ and $\Delta y$ are the spatial steps, $N=[T / \Delta t]$. We will adopt the staggered meshes discretizing (1) - (7).

By discretizing (1) at the reference point $\left(x_{i}, y_{j}, t_{n}\right)$, we obtain

$$
\frac{u_{i+\frac{1}{2}, j}^{n+1}-u_{i-\frac{1}{2}, j}^{n+1}}{\Delta x}+\frac{v_{i, j+\frac{1}{2}}^{n+1}-v_{i, j-\frac{1}{2}}^{n+1}}{\Delta y}=0 .
$$

By discretizing (2) at the reference point $\left(x_{i+1 / 2}, y_{j}, t_{n}\right)$, we obtain

$$
\begin{gathered}
\frac{u_{i+\frac{1}{2}, j}^{n+1}-u_{i+\frac{1}{2}, j}^{n}}{\Delta t}=\frac{\left(F_{1,1}^{2}+F_{1,2}^{2}\right)_{i+1, j}^{n}-\left(F_{1,1}^{2}+F_{1,2}^{2}\right)_{i, j}^{n}}{\Delta x} \\
+\frac{\left(F_{1,1} F_{2,1}+F_{1,2} F_{2,2}\right)_{i+\frac{1}{2}, j+\frac{1}{2}}^{n}-\left(F_{1,1} F_{2,1}+F_{1,2} F_{2,2}\right)_{i+\frac{1}{2}, j-\frac{1}{2}}^{n}}{\Delta y} \\
-\frac{\left(u^{2}\right)_{i+1, j}^{n}-\left(u^{2}\right)_{i, j}^{n}}{\Delta x}+\frac{u_{i+\frac{3}{2}, j}^{n}-2 u_{i+\frac{1}{2}, j}^{n}+u_{i-\frac{1}{2}, j}^{n}}{R e \Delta x^{2}} \\
+\frac{u_{i+\frac{1}{2}, j+1}^{n}-2 u_{i+\frac{1}{2}, j}^{n}+u_{i+\frac{1}{2}, j-1}^{n}}{R e \Delta y^{2}}-\frac{p_{i+1, j}^{n+1}-p_{i, j}^{n}}{\Delta x} \\
-\frac{(u v)_{i+\frac{1}{2}, j+\frac{1}{2}}^{n}-(u v)_{i+\frac{1}{2}, j-\frac{1}{2}}^{n}}{\Delta y}
\end{gathered}
$$

By discretizing (3) at the reference point $\left(x_{i}, y_{j+1 / 2}, t_{n}\right)$, 
we obtain

$$
\begin{aligned}
& \frac{v_{i, j+\frac{1}{2}}^{n+1}-v_{i, j+\frac{1}{2}}^{n}}{\Delta t}=\frac{\left(F_{2,1}^{2}+F_{2,2}^{2}\right)_{i, j+1}^{n}-\left(F_{2,1}^{2}+F_{2,2}^{2}\right)_{i, j}^{n}}{\Delta y} \\
& +\frac{\left(F_{1,1} F_{2,1}+F_{1,2} F_{2,2}\right)_{i+\frac{1}{2}, j+\frac{1}{2}}^{n}-\left(F_{1,1} F_{2,1}+F_{1,2} F_{2,2}\right)_{i-\frac{1}{2}, j+\frac{1}{2}}^{n}}{\Delta x} \\
& -\frac{\left(v^{2}\right)_{i, j+1}^{n}-\left(v^{2}\right)_{i, j}^{n}}{\Delta y}+\frac{v_{i+1, j+\frac{1}{2}}^{n}-2 v_{i, j+\frac{1}{2}}^{n}+u_{i-1, j+\frac{1}{2}}^{n}}{\operatorname{Re} \Delta x^{2}} \\
& +\frac{v_{i, j+\frac{3}{2}}^{n}-2 v_{i, j+\frac{1}{2}}^{n}+v_{i, j-\frac{1}{2}}^{n}}{\operatorname{Re} \Delta y^{2}}-\frac{p_{i+1, j}^{n+1}-p_{i, j}^{n}}{\Delta y} \\
& -\frac{(u v)_{i+\frac{1}{2}, j+\frac{1}{2}}^{n}-(u v)_{i+\frac{1}{2}, j-\frac{1}{2}}^{n}}{\Delta y}
\end{aligned}
$$

By discretizing (4) - (7) at the reference point $\left(x_{i}, y_{j}, t_{n}\right)$, we obtain

$$
\begin{aligned}
& \frac{F_{1,1, i, j}^{n+1}-F_{1,1, i, j}^{n}}{\Delta t}=-\frac{\left(u F_{1,1}\right)_{i+\frac{1}{2}, j}^{n}-\left(u F_{1,1}\right)_{i-\frac{1}{2}, j}^{n}}{\Delta x} \\
& -\frac{\left(v F_{1,1}\right)_{i, j+\frac{1}{2}}^{n}-\left(v F_{1,1}\right)_{i, j-\frac{1}{2}}^{n}}{\Delta y} \\
& +\frac{F_{1,1, i, j}^{n+1} u_{i+\frac{1}{2}, j}^{n}-F_{1,1, i, j}^{n+1} u_{i-\frac{1}{2}, j}^{n}}{\Delta x} \\
& +\frac{F_{2,1, i j}^{n+1} u_{i, j+\frac{1}{2}}^{n}-F_{2,1, i, j}^{n+1} u_{i, j-\frac{1}{2}}^{n}}{\Delta y} \\
& \frac{F_{1,2, i, j}^{n+1}-F_{1,2, i, j}^{n}}{\Delta t}=-\frac{\left(u F_{1,2}\right)_{i+\frac{1}{2}, j}^{n}-\left(u F_{1,2}\right)_{i-\frac{1}{2}, j}^{n}}{\Delta x} \\
& -\frac{\left(v F_{1,2}\right)_{i, j+\frac{1}{2}}^{n}-\left(v F_{1,2}\right)_{i, j-\frac{1}{2}}^{n}}{\Delta y} \\
& +\frac{F_{1,1,2, j}^{n+1} u_{i+\frac{1}{2}, j}^{n}-F_{1,2, i, j}^{n+1} u_{i-\frac{1}{2}, j}^{n}}{\Delta x} \\
& +\frac{F_{2,2, i j}^{n+1} u_{i, j+\frac{1}{2}}^{n}-F_{2,2, i, j}^{n+1} u_{i, j-\frac{1}{2}}^{n}}{\Delta y} \\
& \frac{F_{2,1, i, j}^{n+1}-F_{2,1, i, j}^{n}}{\Delta t}=-\frac{\left(u F_{2,1}\right)_{i+\frac{1}{2}, j}^{n}-\left(u F_{2,1}\right)_{i-\frac{1}{2}, j}^{n}}{\Delta x} \\
& -\frac{\left(v F_{2,1}\right)_{i, j+\frac{1}{2}}^{n}-\left(v F_{2,1}\right)_{i, j-\frac{1}{2}}^{n}}{\Delta y} \\
& +\frac{F_{1,1, i, j}^{n+1} v_{i+\frac{1}{2}, j}^{n}-F_{1,1, i, j}^{n+1} v_{i-\frac{1}{2}, j}^{n}}{\Delta x} \\
& +\frac{F_{2,1, i j}^{n+1} v_{i, j+\frac{1}{2}}^{n}-F_{2,1, i, j}^{n+1} v_{i, j-\frac{1}{2}}^{n}}{\Delta y}
\end{aligned}
$$

$$
\begin{aligned}
\frac{F_{2,2, i, j}^{n+1}-F_{2,2, i, j}^{n}}{\Delta t}= & -\frac{\left(u F_{2,2}\right)_{i+\frac{1}{2}, j}^{n}-\left(u F_{2,2}\right)_{i-\frac{1}{2}, j}^{n}}{\Delta x} \\
& -\frac{\left(v F_{2,2}\right)_{i, j+\frac{1}{2}}^{n}-\left(v F_{2,2}\right)_{i, j-\frac{1}{2}}^{n}}{\Delta y} \\
& +\frac{F_{1,2, i, j}^{n+1} v_{i+\frac{1}{2}, j}^{n}-F_{1,2, i, j}^{n+1} v_{i-\frac{1}{2}, j}^{n}}{\Delta x} \\
& +\frac{F_{2,2, i, j}^{n+1} v_{i, j+\frac{1}{2}}^{n}-F_{2,2, i, j}^{n+1} v_{i, j-\frac{1}{2}}^{n}}{\Delta y},
\end{aligned}
$$

where $i=0,1,2, \ldots, I=\max _{\left(x_{1}, y\right),\left(x_{2}, y\right) \in \Omega}\left|x_{1}-x_{2}\right|, j=$ $0,1,2, \ldots, J=\max _{\left(x, y_{1}\right),\left(x, y_{2}\right) \in \Omega}\left|y_{1}-y_{2}\right|$, and $n=0,1,2$, ..., $N-1$.

Inserting (11) and (12) into (10) could obtain the discrete FD scheme of Poisson equation for $p$ :

$$
\frac{p_{i+1, j}^{n}-2 p_{i, j}^{n}+p_{i-1, j}^{n}}{\Delta x^{2}}+\frac{p_{i, j+1}^{n}-2 p_{i, j}^{n}+p_{i, j-1}^{n}}{\Delta x^{2}}=R^{n},
$$

where $n=0,1,2, \ldots, N, \quad R^{n}=\Delta t\left(\hat{u}_{i+\frac{1}{2}, j}^{n}-\hat{u}_{i-\frac{1}{2}, j}^{n}+\right.$ $\left.\hat{v}_{i, j+\frac{1}{2}}^{n}-\hat{v}_{i, j-\frac{1}{2}}^{n}\right)$, and

$$
\begin{gathered}
\hat{u}_{i+\frac{1}{2}, j}^{n}=\frac{u_{i+\frac{1}{2}, j}^{n}}{\Delta t \Delta x}+\frac{\left(F_{1,1}^{2}+F_{1,2}^{2}\right)_{i+1, j}^{n}-\left(F_{1,1}^{2}+F_{1,2}^{2}\right)_{i, j}^{n}}{\Delta x^{2}} \\
+\frac{\left(F_{1,1} F_{2,1}+F_{1,2} F_{2,2}\right)_{i+\frac{1}{2}, j+\frac{1}{2}}^{n}-\left(F_{1,1} F_{2,1}+F_{1,2} F_{2,2}\right)_{i+\frac{1}{2}, j-\frac{1}{2}}^{n}}{\Delta x \Delta y} \\
-\frac{(u v)_{i+\frac{1}{2}, j+\frac{1}{2}}^{n}-(u v)_{i+\frac{1}{2}, j-\frac{1}{2}}^{n}}{\Delta x \Delta y}+\frac{u_{i+\frac{3}{2}, j}^{n}-2 u_{i+\frac{1}{2}, j}^{n}+u_{i-\frac{1}{2}, j}^{n}}{R e \Delta x^{3}} \\
-\frac{\left(u^{2}\right)_{i+1, j}^{n}-\left(u^{2}\right)_{i, j}^{n}}{\Delta x^{2}}+\frac{u_{i+\frac{1}{2}, j+1}^{n}-2 u_{i+\frac{1}{2}, j}^{n}+u_{i+\frac{1}{2}, j-1}^{n}}{R e \Delta x \Delta y^{2}}, \\
+\frac{\hat{v}_{i, j+\frac{1}{2}}^{n}=\frac{v_{i, j+\frac{1}{2}}^{n}}{\Delta t \Delta y}+\frac{\left(F_{2,1}^{2}+F_{2,2}^{2}\right)_{i, j+1}^{n}-\left(F_{2,1}^{2}+F_{2,2}^{2}\right)_{i, j}^{n}}{\Delta y^{2}}}{\left.\Delta F_{2,1}+F_{1,2} F_{2,2}\right)_{i+\frac{1}{2}, j+\frac{1}{2}}^{n}-\left(F_{1,1} F_{2,1}+F_{1,2} F_{2,2}\right)_{i-\frac{1}{2}, j+\frac{1}{2}}^{n}} \\
-\frac{(u v)_{i+\frac{1}{2}, j+\frac{1}{2}}^{n}-(u v)_{i-\frac{1}{2}, j+\frac{1}{2}}^{n}+\frac{v_{i, j+\frac{3}{2}}^{n}-2 v_{i, j+\frac{1}{2}}^{n}+v_{i, j-\frac{1}{2}}^{n}}{\Delta x \Delta y}}{R e \Delta x^{3}} \\
-\frac{\left(v^{2}\right)_{i, j+1}^{n}-\left(v^{2}\right)_{i, j}^{n}}{\Delta y^{2}}+\frac{v_{i+1, j+\frac{1}{2}}^{n}-2 v_{i, j+\frac{1}{2}}^{n}+v_{i-1, j+\frac{1}{2}}^{n}}{R e \Delta y \Delta x^{2}}
\end{gathered}
$$

Remark 1. The classical FD scheme (11) - (17) is only first-order accurate in time. If one wants to obtain higher order time approximate accuracy, it is necessary to change the time difference coefficients of the left hand sides in the FD scheme (10) - (16) into higher order ones (for example, time central difference coefficients or time second-order difference coefficients). Moreover, because the FD scheme 
(10) - (16) are equivalent to the FD scheme (11) - (17), so we need only to solve the FD scheme (11) - (17). The procedures of solving the FD scheme $(11)-(17)$ are to solve first (17), and then (11) - (12) and (13) - (16).

\section{Stability, Convergence, and Error of FD Solutions}

In this section, we devote ourselves to analyzing the stability, convergence, and errors of the FD solutions for the FD scheme (11) - (17).

Theorem 1. If the temporal step $\Delta t$ and the spatial steps $\Delta x$ and $\Delta y$ satisfy $4 \Delta t \leq \min \left\{\operatorname{Re} \Delta x^{2}, \operatorname{Re} \Delta y^{2}\right\}$, and the initial values $u^{0}(x, y), v^{0}(x, y), F_{l, m}^{0}(x, y) \in L^{\infty}(\Omega)(l, m=1,2)$, i.e., $\left\|u^{0}\right\|_{\infty},\left\|v^{0}\right\|_{\infty},\left\|F_{l, m}^{0}\right\|_{\infty}(l, m=1,2)$ are bounded, then the FD solutions $u_{i+1 / 2, j}^{n+1}, v_{i, j+1 / 2}^{n+1}, p_{i, j}^{n+1}$, and $F_{l, m, i, j}^{n+1}(l, m=$ $1,2)$ for the FD scheme (11) - (17) are stable and convergent.

Proof. By taking absolute value for (13), we obtain

$$
\begin{aligned}
\left|F_{1,1, i, j}^{n+1}\right| \leq & \left|F_{1,1, i, j}^{n}\right|+\frac{\Delta t}{\Delta x}\left|\left(u F_{1,1}\right)_{i+\frac{1}{2}, j}^{n}-\left(u F_{1,1}\right)_{i-\frac{1}{2}, j}^{n}\right| \\
& +\frac{\Delta t}{\Delta y}\left|\left(v F_{1,1}\right)_{i, j+\frac{1}{2}}^{n}-\left(v F_{1,1}\right)_{i, j-\frac{1}{2}}^{n}\right| \\
& +\frac{\Delta t}{\Delta x}\left|F_{1,1, i, j}^{n+1} u_{i+\frac{1}{2}, j}^{n}-F_{1,1, i, j}^{n+1} u_{i-\frac{1}{2}, j}^{n}\right| \\
& +\frac{\Delta t}{\Delta y}\left|F_{2,1, i, j}^{n+1} u_{i, j+\frac{1}{2}}^{n}-F_{2,1, i, j}^{n+1} u_{i, j-\frac{1}{2}}^{n}\right| \\
\leq & \left.+\frac{4 \Delta t}{\Delta x}\left\|u^{n}\right\|_{\infty}+\frac{2 \Delta t}{\Delta y}\left\|v^{n}\right\|_{\infty}\right)\left\|F_{1,1}^{n}\right\|_{\infty} \\
& +\frac{2 \Delta t}{\Delta y}\left\|u^{n}\right\|_{\infty}\left\|F_{2,1}^{n}\right\|_{\infty}
\end{aligned}
$$

where $\|\cdot\|_{\infty}$ represent the norm in $L^{\infty}(\Omega)$. Further, from (18), we obtain

$$
\begin{aligned}
\left\|F_{1,1}^{n+1}\right\|_{\infty} \leq( & \left.+\frac{4 \Delta t}{\Delta x}\left\|u^{n}\right\|_{\infty}+\frac{2 \Delta t}{\Delta y}\left\|v^{n}\right\|_{\infty}\right)\left\|F_{1,1}^{n}\right\|_{\infty} \\
& +\frac{2 \Delta t}{\Delta y}\left\|u^{n}\right\|_{\infty}\left\|F_{2,1}^{n}\right\|_{\infty}
\end{aligned}
$$

By using the same approaches as the proof of (19), from (14)-(16), we obtain

$$
\begin{aligned}
\left\|F_{1,2}^{n+1}\right\|_{\infty} \leq & \left.+\frac{4 \Delta t}{\Delta x}\left\|u^{n}\right\|_{\infty}+\frac{2 \Delta t}{\Delta y}\left\|v^{n}\right\|_{\infty}\right)\left\|F_{1,2}^{n}\right\|_{\infty} \\
+ & \frac{2 \Delta t}{\Delta y}\left\|u^{n}\right\|_{\infty}\left\|F_{2,2}^{n}\right\|_{\infty} \\
\left\|F_{2,1}^{n+1}\right\|_{\infty} \leq( & \left.+\frac{2 \Delta t}{\Delta x}\left\|u^{n}\right\|_{\infty}+\frac{4 \Delta t}{\Delta y}\left\|v^{n}\right\|_{\infty}\right)\left\|F_{2,1}^{n}\right\|_{\infty} \\
& +\frac{2 \Delta t}{\Delta x}\left\|v^{n}\right\|_{\infty}\left\|F_{2,1}^{n}\right\|_{\infty}
\end{aligned}
$$

$$
\begin{gathered}
\left\|F_{2,2}^{n+1}\right\|_{\infty} \leq\left(1+\frac{2 \Delta t}{\Delta x}\left\|u^{n}\right\|_{\infty}+\frac{4 \Delta t}{\Delta y}\left\|v^{n}\right\|_{\infty}\right)\left\|F_{2,2}^{n}\right\|_{\infty} \\
+\frac{2 \Delta t}{\Delta x}\left\|v^{n}\right\|_{\infty}\left\|F_{1,2}^{n}\right\|_{\infty}
\end{gathered}
$$

By combining (19) with (21) and (20) with (22), we obtain

$$
\begin{gathered}
\left\|F_{1,1}^{n}\right\|_{\infty}+\left\|F_{2,1}^{n}\right\|_{\infty} \leq\left(\left\|F_{1,1}^{n-1}\right\|_{\infty}+\left\|F_{2,1}^{n-1}\right\|_{\infty}\right) \\
\times\left(1+\frac{4 \Delta t}{\Delta x}\left\|u^{n-1}\right\|_{\infty}+\frac{4 \Delta t}{\Delta y}\left\|v^{n-1}\right\|_{\infty}\right), \\
\left\|F_{1,2}^{n}\right\|_{\infty}+\left\|F_{2,2}^{n}\right\|_{\infty} \leq\left(\left\|F_{1,2}^{n-1}\right\|_{\infty}+\left\|F_{2,2}^{n-1}\right\|_{\infty}\right) \\
\times\left(1+\frac{4 \Delta t}{\Delta x}\left\|u^{n-1}\right\|_{\infty}+\frac{4 \Delta t}{\Delta y}\left\|v^{n-1}\right\|_{\infty}\right) .
\end{gathered}
$$

By summing (23) and (24) from 1 to $n$, we obtain

$$
\begin{array}{r}
\left\|F_{1,1}^{n}\right\|_{\infty}+\left\|F_{2,1}^{n}\right\|_{\infty} \leq\left\|F_{1,1}^{0}\right\|_{\infty}+\left\|F_{2,1}^{0}\right\|_{\infty}+ \\
4 \Delta t \sum_{k=0}^{n-1}\left(\frac{\left\|u^{k}\right\|_{\infty}}{\Delta x}+\frac{\left\|v^{k}\right\|_{\infty}}{\Delta y}\right)\left(\left\|F_{1,1}^{k}\right\|_{\infty}+\left\|F_{2,1}^{k}\right\|_{\infty}\right), \\
\left\|F_{1,2}^{n}\right\|_{\infty}+\left\|F_{2,2}^{n}\right\|_{\infty} \leq\left\|F_{1,2}^{0}\right\|_{\infty}+\left\|F_{2,2}^{0}\right\|_{\infty}+ \\
4 \Delta t \sum_{k=0}^{n-1}\left(\frac{\left\|u^{k}\right\|_{\infty}}{\Delta x}+\frac{\left\|v^{k}\right\|_{\infty}}{\Delta y}\right)\left(\left\|F_{1,2}^{k}\right\|_{\infty}+\left\|F_{2,2}^{k}\right\|_{\infty}\right)
\end{array}
$$

If the condition $4 \Delta t \leq \min \left\{\operatorname{Re} \Delta x^{2}, \operatorname{Re} \Delta y^{2}\right\}$ is satisfied, then, from (11), we have

$$
\begin{aligned}
& \left|u_{i+\frac{1}{2}, j}^{n+1}\right| \leq\left(1+\frac{2 \Delta t}{R e \Delta x^{2}}+\frac{2 \Delta t}{R e \Delta y^{2}}\right)\left|u_{i+\frac{1}{2}, j}^{n}\right| \\
& +\frac{\Delta t}{R e \Delta x^{2}}\left(\left|u_{i+\frac{3}{2}, j}^{n}\right|+\left|u_{i-\frac{1}{2}, j}^{n}\right|\right)+\frac{\Delta t}{\Delta x}\left(\left|u_{i+1, j}^{n}\right|^{2}+\left|u_{i, j}^{n}\right|^{2}\right) \\
& +\frac{\Delta t}{R e \Delta y^{2}}\left(\left|u_{i+\frac{1}{2}, j+1}^{n}\right|+\left|u_{i+\frac{1}{2}, j-1}^{n}\right|\right)+\frac{\Delta t}{\Delta x}\left(\left|p_{i+1, j}^{n}\right|+\left|p_{i, j}^{n}\right|\right) \\
& +\frac{\Delta t}{\Delta x}\left(\left|F_{1,1, i+1, j}^{n}\right|^{2}+\left|F_{1,2, i+1, j}^{n}\right|^{2}+\left|F_{1,1, i, j}^{n}\right|^{2}+\left|F_{1,2, i, j}^{n}\right|^{2}\right) \\
& +\frac{\Delta t}{\Delta y}\left(\left|\left(F_{1,1} F_{2,1}+F_{12} F_{2,2}\right)_{i+\frac{1}{2}, j+\frac{1}{2}}^{n}\right|\right. \\
& \left.+\left|\left(F_{1,1} F_{2,1}+F_{12} F_{2,2}\right)_{i+\frac{1}{2}, j-\frac{1}{2}}^{n}\right|\right) \\
& +\frac{\Delta t}{\Delta y}\left(\left|(u v)_{i+\frac{1}{2}, j+\frac{1}{2}}^{n}\right|+\left|(u v)_{i+\frac{1}{2}, j-\frac{1}{2}}^{n}\right|\right) \\
& \leq\left\|u^{n}\right\|_{\infty}+\frac{2 \Delta t}{\Delta x}\left\|u^{n}\right\|_{\infty}^{2} \\
& +\frac{2 \Delta t}{\Delta y}\left\|u^{n}\right\|_{\infty}\left\|v^{n}\right\|_{\infty} \\
& +\frac{2 \Delta t}{\Delta y}\left(\left\|F_{1,1}^{n}\right\|_{\infty}\left\|F_{2,1}^{n}\right\|_{\infty}+\left\|F_{1,2}^{n}\right\|_{\infty}\left\|F_{2,2}^{n}\right\|_{\infty}\right) \\
& +\frac{2 \Delta t}{\Delta x}\left(\left\|F_{1,1}^{n}\right\|_{\infty}^{2}+\left\|F_{2,2}^{n}\right\|_{\infty}^{2}+\left\|p^{n}\right\|_{\infty}\right) .
\end{aligned}
$$

Further, we have 


$$
\begin{gathered}
\left\|u^{n+1}\right\|_{\infty} \leq\left\|u^{n}\right\|_{\infty}+\frac{2 \Delta t}{\Delta x}\left\|u^{n}\right\|_{\infty}^{2}+\frac{2 \Delta t}{\Delta y}\left\|u^{n}\right\|_{\infty}\left\|v^{n}\right\|_{\infty} \\
+\frac{2 \Delta t}{\Delta y}\left(\left\|F_{1,1}^{n}\right\|_{\infty}\left\|F_{2,1}^{n}\right\|_{\infty}+\left\|F_{1,2}^{n}\right\|_{\infty}\left\|F_{2,2}^{n}\right\|_{\infty}\right) \\
+\frac{2 \Delta t}{\Delta x}\left(\left\|F_{1,1}^{n}\right\|_{\infty}^{2}+\left\|F_{2,2}^{n}\right\|_{\infty}^{2}+\left\|p^{n}\right\|_{\infty}\right),
\end{gathered}
$$

where $n=0,1,2, \ldots, N-1$. By using the same approaches as the proof of (27), from (12), we obtain

$$
\begin{aligned}
\left\|v^{n+1}\right\|_{\infty} & \leq\left\|v^{n}\right\|_{\infty}+\frac{2 \Delta t}{\Delta y}\left\|v^{n}\right\|_{\infty}^{2}+\frac{2 \Delta t}{\Delta x}\left\|u^{n}\right\|_{\infty}\left\|v^{n}\right\|_{\infty} \\
+ & \frac{2 \Delta t}{\Delta x}\left(\left\|F_{1,1}^{n}\right\|_{\infty}\left\|F_{2,1}^{n}\right\|_{\infty}+\left\|F_{1,2}^{n}\right\|_{\infty}\left\|F_{2,2}^{n}\right\|_{\infty}\right) \\
+ & \frac{2 \Delta t}{\Delta y}\left(\left\|F_{2,1}^{n}\right\|_{\infty}^{2}+\left\|F_{2,2}^{n}\right\|_{\infty}^{2}+\left\|p^{n}\right\|_{\infty}\right),
\end{aligned}
$$

where $n=0,1,2, \ldots, N-1$.

Se $\boldsymbol{p}=\left(p_{0,0}, p_{1,0}, \ldots, p_{I, 0}, p_{0,1}, p_{1,1}, \ldots, p_{I, 1}, \ldots, p_{0, J}, p_{1, J}, \ldots\right.$, $\left.p_{I, J}\right)^{T}$. Then, we can rewrite (17) into the following vector form:

$$
\left(\frac{\boldsymbol{B}}{\Delta x^{2}}+\frac{\boldsymbol{C}}{\Delta y^{2}}\right) \boldsymbol{p}^{n}=\widetilde{\boldsymbol{R}}^{n}, n=0,1,2, \ldots, N,
$$

where the vectors $\widetilde{\boldsymbol{R}}^{n}$ 's are determined by $R^{n}$ in (17) and $\boldsymbol{B}$ and $\boldsymbol{C}$ are, respectively, two $[(I+1) \times(J+1)]$-order symmetrical matrices as follows:

$$
\begin{aligned}
& \boldsymbol{B}=\left(\begin{array}{cccccc}
-2 & 1 & 0 & \cdots & 0 & 0 \\
1 & -2 & 1 & \cdots & 0 & 0 \\
0 & 1 & -2 & \ddots & 0 & 0 \\
\vdots & \vdots & \ddots & \ddots & \ddots & \vdots \\
0 & 0 & 0 & \ddots & -2 & 1 \\
0 & 0 & 0 & \cdots & 1 & -2
\end{array}\right),
\end{aligned}
$$

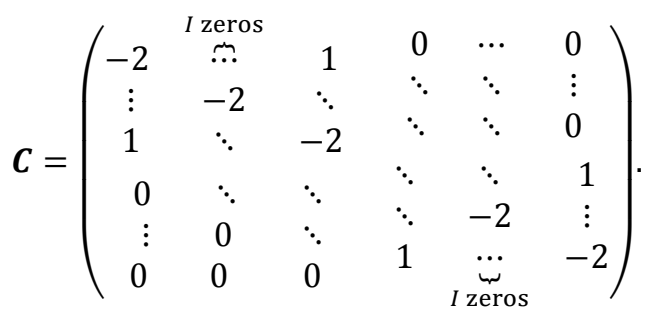

It is obvious that the matrix $\left(\boldsymbol{B} / \Delta x^{2}+\boldsymbol{C} / \Delta y^{2}\right)$ is reversible and the maximum norm of its reverse matrix is bounded. Note that the maximum value of the absolute value of each component of vector is no more than its maximum norm.

$$
\left\|p^{n}\right\|_{\infty} \leq\left\|\boldsymbol{p}^{n}\right\|_{\infty} \leq\left\|\left(\boldsymbol{B} / \Delta x^{2}+\boldsymbol{C} / \Delta y^{2}\right)^{-1}\right\|_{\infty}\left\|\widetilde{\boldsymbol{R}}^{n}\right\|_{\infty} .
$$

Therefore, when $\left\|u^{0}\right\|_{\infty},\left\|v^{0}\right\|_{\infty},\left\|F_{l, m}^{0}\right\|_{\infty}(l, m=1,2)$ are bounded, then $\left\|p^{0}\right\|_{\infty}$ are also bounded. Further, from (25) to (29), we can recurrently deduce $\left\|u^{n}\right\|_{\infty},\left\|v^{n}\right\|_{\infty},\left\|p^{n}\right\|_{\infty}$, and $\left\|F_{l, m}^{n}\right\|_{\infty}(l, m=1,2, n=1,2, \ldots, N)$ all are bounded. Thus, by the stability principle (see [15] or [16]), we immediately obtain that the FD solutions $u_{i+1 / 2, j}^{n+1}, v_{i, j+1 / 2}^{n+1}, p_{i, j}^{n+1}$, and $F_{l, m, i, j}^{n+1}(l, m=1,2)$ for the FD scheme (11) - (17) are stable and convergent. This completes the proof of Theorem 1 .

For the solutions of the FD scheme (10) - (16), we have the following error estimates.

Theorem 2. Let $\boldsymbol{U}=(u, v)^{T}, \boldsymbol{F}=\left(F_{l, m}\right)_{2 \times 2}$, and $p$ be the accuracy solution for the system of Eqs (1) - (9). Then the FD solutions $u_{i+1 / 2, j}^{n+1}, v_{i, j+1 / 2}^{n+1}, p_{i, j}^{n+1}$, and $F_{l, m, i, j}^{n+1}(l, m=1,2)$ for the FD scheme (11) - (17) have the following error estimates

$$
\begin{aligned}
& \left|u\left(x_{i+\frac{1}{2}}, y_{j}, t_{n}\right)-u_{i+\frac{1}{2}, j}^{n}\right|+\left|v\left(x_{i}, y_{j+\frac{1}{2}}, t_{n}\right)-v_{i, j+\frac{1}{2}}^{n}\right| \\
+ & \left|p\left(x_{i}, y_{j}, t_{n}\right)-p_{i, j}^{n}\right|+\sum_{l, m=1}^{2}\left|F_{l, m}\left(x_{i}, y_{j}, t_{n}\right)-F_{l, m, i, j}^{n}\right| \\
= & O\left(\Delta t, \Delta x^{2}, \Delta y^{2}\right), 0 \leq n \leq N, 0 \leq i \leq I, 0 \leq j \leq J .
\end{aligned}
$$

Proof. By using Taylor's formula to expand (10) and (13) (16) at the reference point $\left(x_{i}, y_{j}, t_{n}\right),(11)$ at the reference point $\left(x_{i+1 / 2}, y_{j}, t_{n}\right)$, (12) at the reference point $\left(x_{i}, y_{j+1 / 2}, t_{n}\right)$, or from the discretizing procedure of (10) (16), we could obtain the following error estimates (30). This completes the proof of Theorem 2 .

\section{Some Numerical Experiments}

In this section, some numerical experiments are provided to verify the validity of the FD scheme for the 2D incompressible nonlinear viscoelastic fluid dynamics problem, i.e., the system of Eqs (1) - (9).

In the 2D incompressible nonlinear viscoelastic fluid dynamics problem, i.e., the system of Eqs (1) - (9), we chose the computational domain as $\Omega=\{(x, y): 0 \leq x \leq 50,30 \leq$ $y \leq 70\} \cup\{(x, y): 50 \leq x \leq 100,0 \leq y \leq 100\}, \operatorname{Re}=1000$, and the initial and boundary values of the fluid velocity $U=(u, v)^{T} \quad$ as $\quad\left(u^{0}, v^{0}\right)=\left(u_{0}, v_{0}\right)=(2(y-30)(70-$ $y), 0)(x=0,30 \leq y \leq 70) \quad$ and $\left(u^{0}, v^{0}\right)=\left(u_{0}, v_{0}\right)$ satisfying that $\partial u_{0} / \partial x=p R e$ and $v_{0}=0$ on $\{(x, y): x=$ $100,0 \leq y \leq 100\}$, but $\left(u^{0}, v^{0}\right)=\left(u_{0}, v_{0}\right)=(0,0)$ on other solid boundaries. Whereas the initial and boundary values of the deformation tensor $F=\left(F_{l, m}\right)_{2 \times 2}$ satisfied that $F_{1,1}=$ $F_{2,2}=1$ and $F_{1,2}=F_{2,1}=0$. In additional, we chose $\Delta x=\Delta y$ $=\Delta t=0.01$ in order to satisfy $4 \mu \Delta t \leq \min \left\{\Delta x^{2}, \Delta y^{2}\right\}$. We operated the FD scheme (11) - (17) to obtain the numerical solutions $U=(u, v)^{T}, p, F_{1,1}, F_{1,2}, F_{2,1}$, and $F_{2,2}$ at $t=10$, which were still convergent and were drawn in the photos in Figures. 1 to 6 , respectively. These photos showed that the FD scheme can effectively simulate the physical problems.

Figures. 7 and 8 expressed the relative errors of the FD solutions of the fluid velocity $U=(u, v)^{T}$ and the pressure $p$ as well as the deformation tensor $F=\left(F_{l, m}\right)_{2 \times 2}$ on $0 \leq t \leq 10$, respectively. The variation tendencies of the error curves were consistent with the true situations because the relative errors of the FD solutions gradually increase as the truncation errors were accumulated in the calculating procedure. These relative error 
curves also expressed that the numerical simulating errors were consistent with the theoretical ones because the theoretical and numerical errors all were $O\left(10^{-2}\right)$. This implies that the FD scheme is very effective for finding the $2 \mathrm{D}$ incompressible nonlinear viscoelastic fluid dynamics problem.

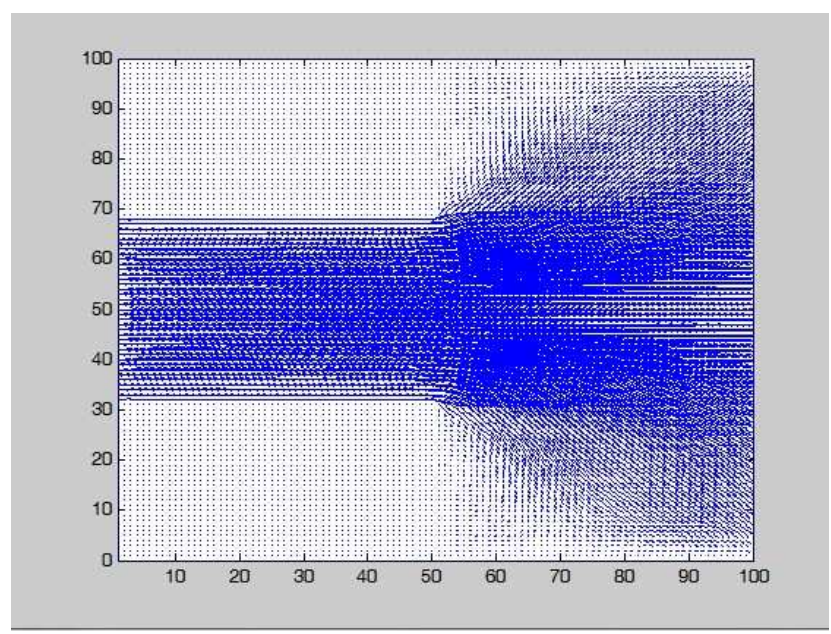

Figure 1. The streamline of the FD solution of the fluid velocity $U$ at $t=10$.

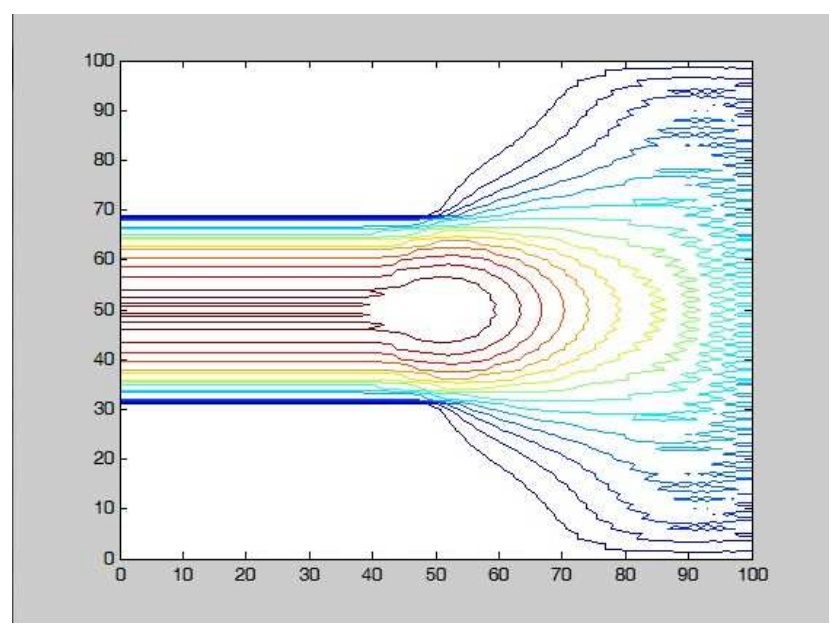

Figure 2. The FD solution of the pressure $p$ at $t=10$.

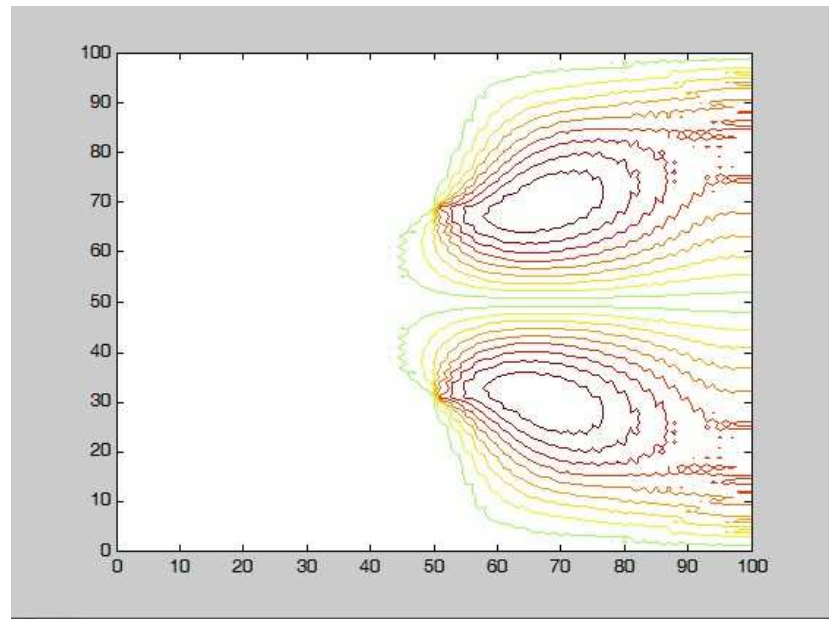

Figure 3. The FD solution of the component $F_{1,1}$ of the deformation tensor $F=\left(F_{l, m}\right)_{2 \times 2}$ at $t=10$.

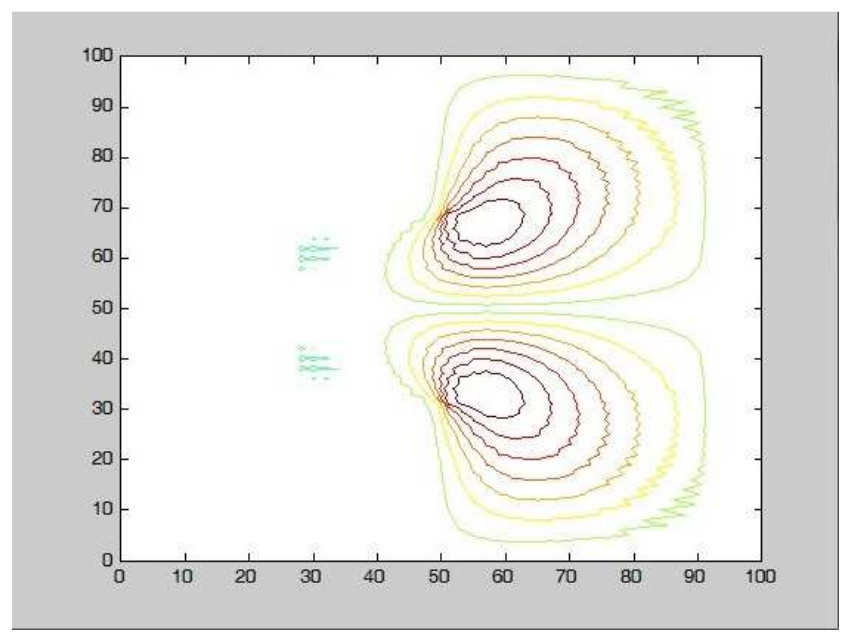

Figure 4. The FD solution of the component $F_{1,2}$ of the deformation tensor $F=\left(F_{l, m}\right)_{2 \times 2}$ at $t=10$.

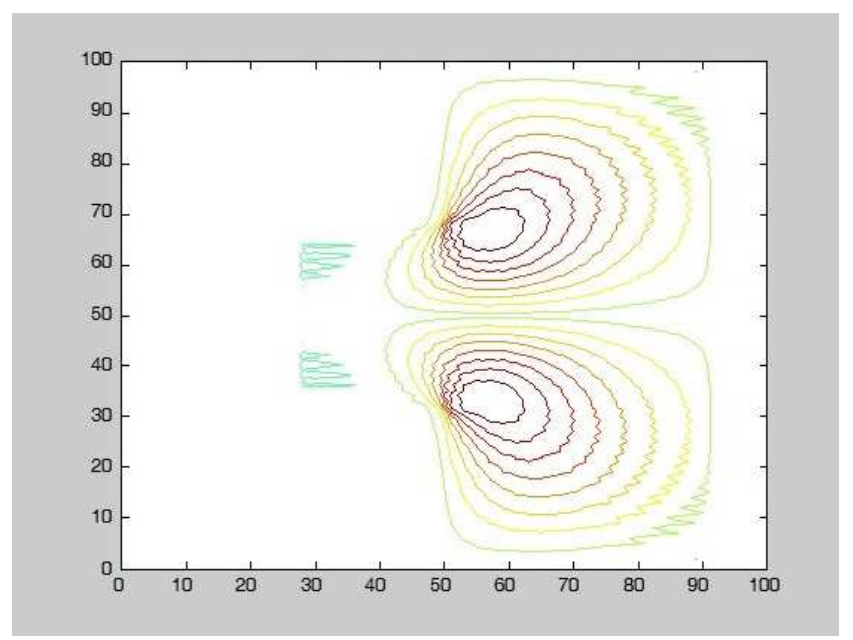

Figure 5. The FD solution of the component $F_{2,1}$ of the deformation tensor $F=\left(F_{l, m}\right)_{2 \times 2}$ at $t=10$.

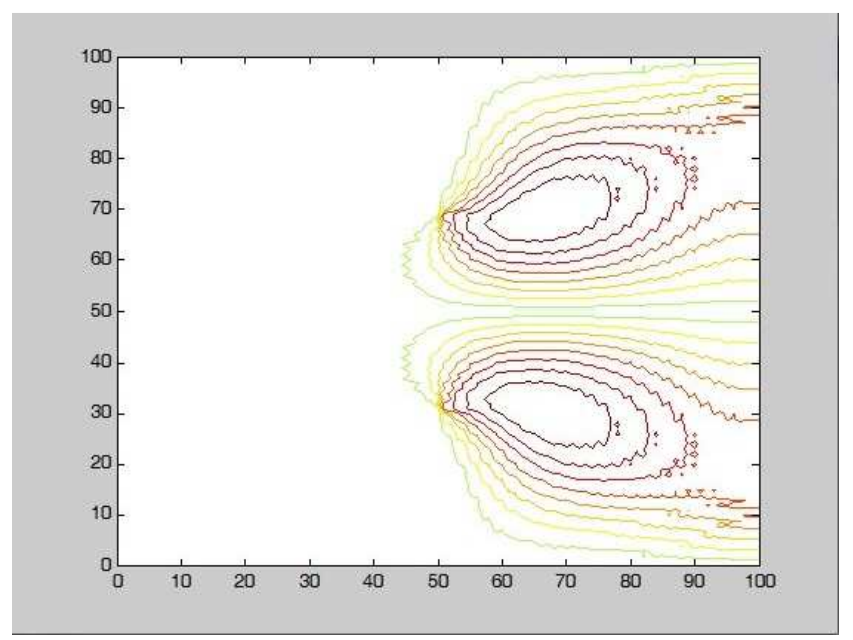

Figure 6. The FD solution of the component $F_{2,2}$ of the deformation tensor $F=\left(F_{l, m}\right)_{2 \times 2}$ at $t=10$. 


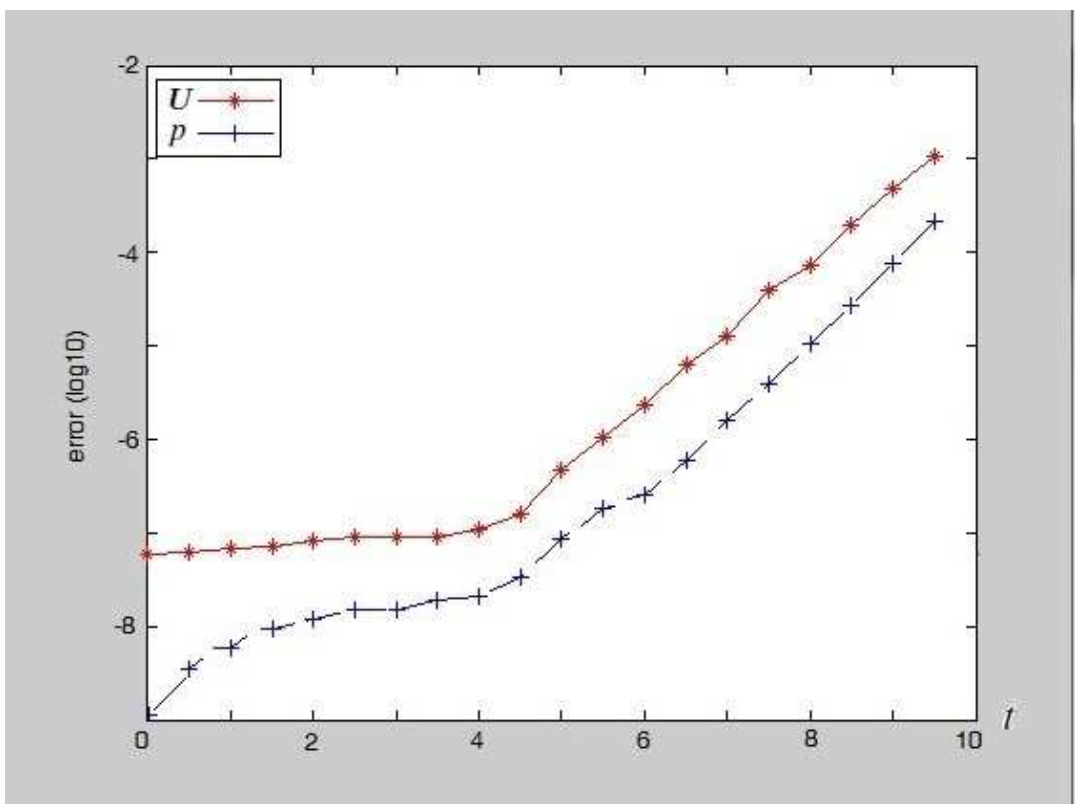

Figure 7. The relative error trending charts of the FD solutions of the fluid velocity $U$ and the pressure $p$ on $0 \leq t \leq 10$.

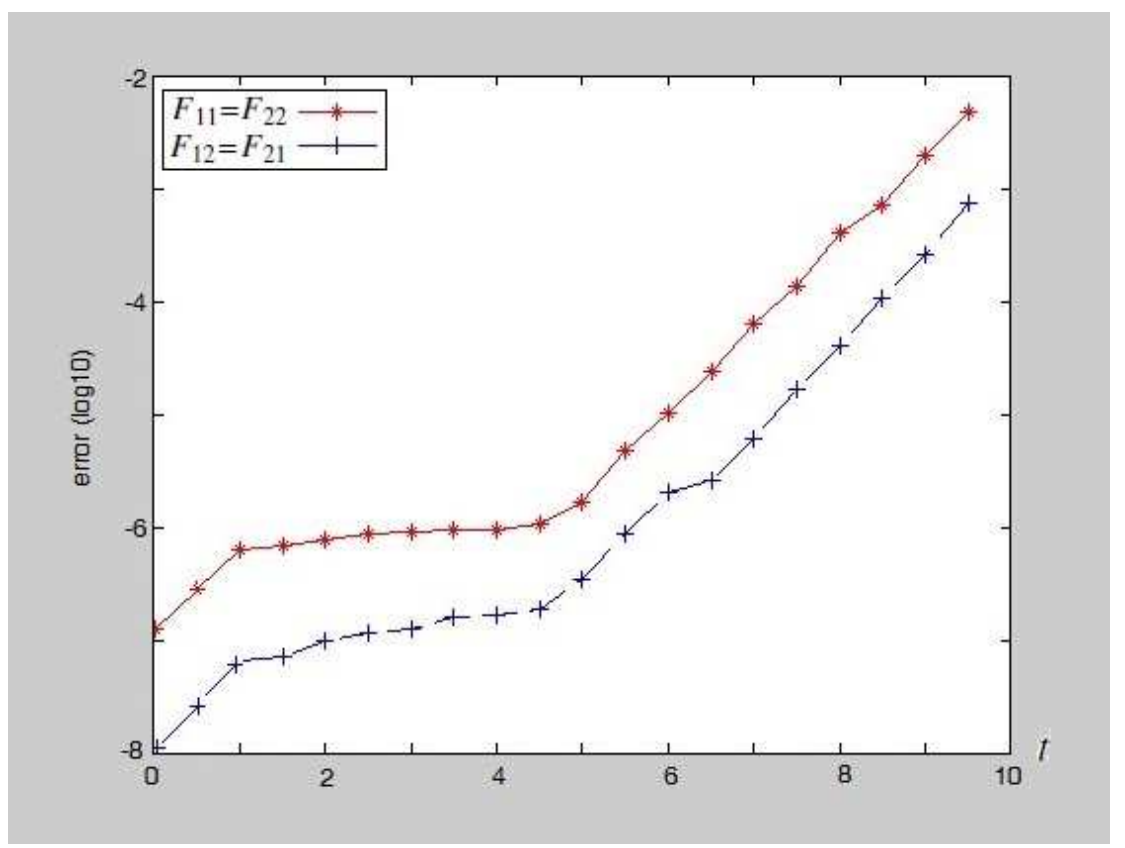

Figure 8. The relative error trending charts of the FD solutions of the components of the deformation tensor $F=\left(F_{l, m}\right)_{2 \times 2}$ on $0 \leq t \leq 10$.

\section{Conclusions and Discussions}

In this article, we have built the stable and convergent FD scheme on the staggered meshes for the 2D incompressible nonlinear viscoelastic fluid dynamics problem, i.e., the system of Eqs (1) - (9). We have also offered the theoretical analysis about the stability, convergence, and errors of the FD solutions and used some numerical experiments to confirm the validity of the FD scheme. This FD scheme is first established because at present there has not been any FD scheme reported for the 2D incompressible nonlinear viscoelastic fluid dynamics problem. This implies that the technique here is development for the existing results (see, e.g., [7-13]).

The 2D incompressible nonlinear viscoelastic fluid dynamics problem, i.e., the system of Eqs (1) - (9) are often used to describe the real-world fluid flow. Therefore, the work in this article has not only the important theoretical value but also holds many real-life applications.

\section{Acknowledgements}

This work is jointly supported by the National Science Foundation of China (11671106 and 11461026). 


\section{References}

[1] A. J. Zhao and D. P. Du, "Local well-posedness of lower regularity solutions for the incompressible viscoelastic fluid system,”Science China Math., 2010, 53(6), 1520-1530.

[2] R. B. Bird, R. C. Armstrong and O. Hassager, "Dynamics of Polymeric Liquids," Volume 1, Fluid Mechanics, Weiley Intersience, New York, 1987.

[3] P. G. de Gennes and J. Prost, "The Physics of Liquid Crystals," Oxford University Press, New York, 1993.

[4] R. G. Larson, "The Structure and Rheology of complex fluids," Oxford University Press, New York, 1995.

[5] T. C. Sideris and B. Thomases, "Global existence for 3D incompressible isotropic elastodynamics via the incompressible limit," Comm. Pure Appl. Math., 2005, 58(6), 750-788.

[6] Z. Lei, C. Liu and Y. Zhou, "Golbal solutions for the incompressible viscoelastic fluids," Arch. Rational Mech. Anal., 2008, 188(3), 371-398.

[7] I. E. Barton and R. Kirby, "Finite difference scheme for the solution of fluid flow problems on non-staggered grids," International Journal for Numerical Methods in Fluids, 2000, 33(7), 939-959.

[8] M. M. Khader and A. M. Megahed, "Numerical simulation using the finite difference method for the flow and heat transfer in a thin liquid film over an unsteady stretching sheet in a saturated porous medium in the presence of thermal radiation," Journal of King Saud University-Engineering Sciences, 2013, 25, 29-34.
[9] S. K. Pandit, J. C. Kalita and D. C. Dalal, "A transient higher order compact scheme for incompressible viscous flows on geometries beyond rectangular," Journal of Computational Physics, 2007, 225(1), 1100-1124.

[10] I. Soukup, "Numerical method based on DGM for solving the system of equations describing motion of viscoelastic fluid with memory," In: Numerical Mathematics and Advanced Applications ENUMATH 2015, B. Karasözen et al. (eds.). Lecture Notes in Computational Science and Engineering, Springer International Publishing Switzerland, 2016, 112, 205-213.

[11] P. A. B. de Sampaio, "A stabilized finite element method for incompressible flow and heat transfer: A natural derivation based on the use of local time-steps," Computer Methods in Applied Mechanics and Engineering, 2006, 195(44-47), 61776190.

[12] P. A. B. de Sampaio, "A finite element formulation for transient incompressible viscous flows stabilized by local time-steps," Computer Methods in Applied Mechanics and Engineering, 2005, 194(18-20), 2095-2108.

[13] C. O. Faria, and J. Karam-Filho, "A regularized-stabilized mixed finite element formulation for viscoplasticity of Bingham type," Computers \& Mathematics with Applications, 2013, 66(6), 975-995.

[14] H. Xia and Z. D. Luo, "Stabilized finite volume element method for the 2D nonlinear incompressible viscoelastic flow equation," Boundary Value Problems, 2017, 2017(130), 1-17.

[15] T. Chung, "Computational Fluid Dynamics", Cambridge University Press, Cambridge, 2002.

[16] R. X. Liu, and C. W. Shu, "Several New Methods for Computational Fluid Mechanics (in Chinese)," Science Press, Beijing, 2003. 